\title{
Plasma fibronectin concentrations in mucocutaneous lymph node syndrome
}

\author{
S SHIMIZU, T KURATSUJI, T OJIMA, AND E TAKAHASHI \\ Division of Pediatrics, Otawara Red Cross Hospital, and Department of Pediatrics, School of Medicine, \\ Keio University
}

SUMMARY Plasma fibronectin concentrations were measured in 41 patients suffering from mucocutaneous lymph node syndrome (MCLS, Kawasaki disease). Plasma fibronectin concentrations were decreased significantly in the early days of the disease, but increased gradually and reached significantly high concentrations by the fourth week of the disease.

In nine of the 41 patients, coronary involvement was found by echocardiographic examination. These patients showed significantly lower plasma fibronectin concentrations in the first and second weeks of the disease than those without coronary involvement.

As the underlying pathology of MCLS is known to be vasculitis, the lower plasma fibronectin concentrations might be partly the result of injury inflicted upon the endothelial cells, which are thought to be the major site of synthesis of circulating plasma fibronectin concentrations.

Fibronectin is an adhesive glycoprotein that is found on many cell surfaces and in extracellular fluids. A wide variety of its properties has been revealed recently. Plasma fibronectin is known to play a role in phagocytosis of neutrophils ${ }^{1}$ and macrophages, ${ }^{2}$ clearance of collagenous debris by the reticuloendothelial system, ${ }^{3}$ wound healing, and so on.

As commercially available kits for the measurement of human plasma fibronectin concentrations are available, we have measured it in several groups of normal and diseased children. ${ }^{4}$ Among them, patients suffering from MCLS seemed to show a distinct trend during the course of their disease.

\section{Materials and methods}

Patients. Forty one patients, who fulfilled the diagnostic criteria for MCLS prepared by the research committee of MCLS, ${ }^{5}$ which was supported by the Ministry of Health and Welfare of the Japanese government, were included in the study. These included 21 boys and 20 girls; the ages of the patients were between 2 months and 4 years. The Table shows the patients' age and sex. Of the 41 patients, nine were shown to have some coronary involvement by echocardiographic examination, which was performed at least once in the convalescent phase, usually twice or more serially.
Table Distribution of patients according to sex and age. (Values in parentheses are No of patients with coronary involvement)

\begin{tabular}{lccc}
\hline Age (yrs) & No & Boys & Girls \\
\hline$<1$ & 11 & $7(3)$ & $4(2)$ \\
$1-<2$ & 14 & $6(1)$ & $8(1)$ \\
$2-<3$ & 7 & $5(1)$ & $2(0)$ \\
$3-<4$ & 7 & $2(1)$ & $5(0)$ \\
$4-<5$ & 2 & $1(0)$ & $1(0)$ \\
\hline Total & 41 & $21(6)$ & $200^{\circ}(3)$ \\
\hline
\end{tabular}

Samples. Blood samples were drawn by venipunc- $\frac{T}{0}$ ture from patients with MCLS. Plasma was separated with sodium citrate and stored at $-40^{\circ} \mathrm{C}$ until $\stackrel{\sim}{\sigma}$ assayed. A total of 165 samples were collected from $N$ the 41 patients. One to 12 samples were obtained N from each patient on days ranging from days 2 to $92 \stackrel{\omega}{\sigma}$ of the disease.

Measurement. A commercially available kit for determination of human plasma fibronectin concentrations, issued by Boehringer Mannheim Yama- ${ }_{0}^{\circ}$ nouchi Co Ltd, was used. The principle of this test is $\overrightarrow{\mathbb{D}}$ a kinetic turbidimetric measurement of the antigenantibody reaction. 
Consent. Consent for the sampling was obtained from all parents of the MCLS patients.

Statistical analysis. The groups were compared using the $t$ test and Welch's test according to the sample distribution.

\section{Results}

Changes in plasma fibronectin concentrations during the days after onset are shown in Figure 1. The shaded area represents the normal range of plasma fibronectin concentrations for infants and children in our hospital. ${ }^{4}$ The decreased plasma fibronectin concentrations in the early days of the disease and the following tendency to increase are obvious. Figure 2 represents the plasma fibronectin concentrations in patients with MCLS classified according to the day of sampling from the onset of disease. The concentration (173 (SD 58) $\mathrm{mg} / \mathrm{l}, \mathrm{n}=24$ ) in the first week of the disease was significantly decreased, and the values at the fourth, sixth, and seventh week or later were significantly increased compared with the normal values in our hospital.

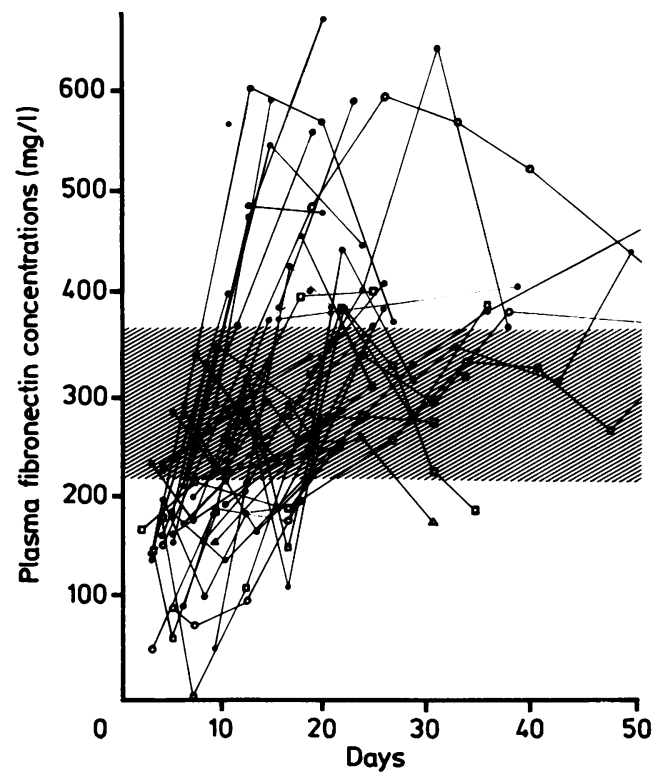

Fig. 1 Serial measurement of plasma fibronectin concentrations in each patient with $M C L S .(-$ without coronary involvement $;-$
$=$ with aneurysm; $\longrightarrow \triangle \longrightarrow=$ with dilatation; $-\square-=$ with irregularity of coronary artery wall in echocardiographic examination.) Shaded area represents the normal range in our hospital.

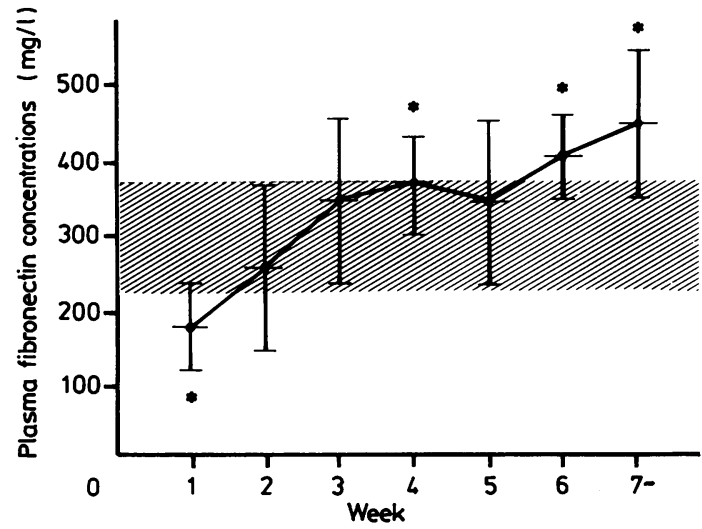

Fig. 2 Plasma fibronectin concentrations in patients with MCLS and date of sampling. Shaded area represents the normal range in our hospital; brackets represent mean $(S D)$.

${ }^{*} \mathrm{p}<0 \cdot 01$.

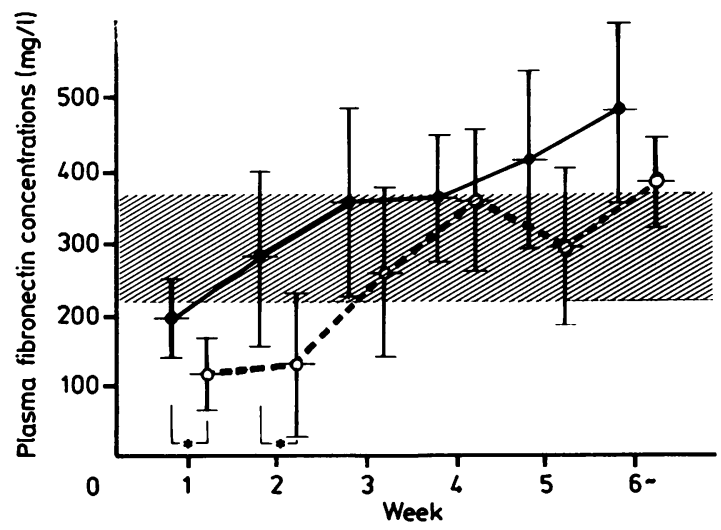

Fig. 3 Plasma fibronectin concentrations in patients with MCLS: comparison between those with (- - $\bigcirc--)$ and without (-) coronary involvement. Shaded area represents the normal range in our hospital; brackets represent mean $(S D)$.

${ }^{*} \mathrm{p}<0 \cdot 01$.

The plasma fibronectin concentrations in patients with MCLS without coronary involvement were higher than those with coronary involvement at any week of the disease that had been measured (Fig. 3). In the first and second week of the disease, particularly, the differences between those with and without coronary involvement were significant. The number of samples included were 109 samples from 32 patients without coronary involvement and 56 
samples from nine patients with coronary involvement.

\section{Discussion}

The cause of MCLS is not yet known, although some bacteria, viruses, and toxins are thought to participate in the pathogenesis of this condition. Plasma fibronectin is known to act as a non-specific opsonin for the reticuloendothelial system. ${ }^{3}$ Accordingly, it is possible to attribute the reduced plasma fibronectin concentrations to its consumption during the clearance of macromolecules such as immune complexes or bacteria. Another reason for the decreased concentrations of plasma fibronectin in the early stage of MCLS, however, may be that, because the underlying pathology of MCLS is vasculitis, ${ }^{6}$ there may be damage to the endothelial cells, which are thought to be a major site of synthesis of plasma fibronectin. ${ }^{7}$ If this is indeed the case, the degree of decrease of the plasma fibronectin concentrations may reasonably correlate with the degree of vasculitis of the coronary artery as well as with the degree of coronary involvement. Plasma fibronectin concentrations are known to be lower in younger individuals, ${ }^{4}$ in whom coronary involvement is more commonly observed, as shown in the Table. It was therefore suspected that the lower plasma fibronectin concentrations in patients with coronary involvement might be due to the age groups concerned. To investigate this possibility, the plasma fibronectin concentrations in patients with and without coronary involvement were compared with those in each age group, and the results confirmed that the concentrations were lower in patients with coronary involvement, irrespective of age (data not shown).

The treatment of MCLS is not yet established. At present, it is important to detect coronary involvement as early as possible. Nowadays, echocardiographic examinations are conveniently available at almost every hospital or clinic, and serial and meticulous follow up study using echocardiographic examination is the standard method of management of MCLS in this country. Unfortunately, coronary involvement cannot be detected with this method until it becomes obvious. It seems valuable, there- $\frac{\tau}{\sigma}$ fore, to anticipate coronary involvement in patients $\frac{C}{0}$ with MCLS. If a low initial fibronectin concentra- $\overline{\frac{\sigma}{\omega}}$ tion is a possible indicator of subsequent formation $\frac{\vec{\sigma}}{\delta}$ of aneurysms the determination of plasma fibro- $\stackrel{\varrho}{\circ}$ nectin concentrations in patients with MCLS will be कs of some clinical use in selecting more severely $\overrightarrow{0}$ affected patients for aggressive treatment. In terms $\vec{\overrightarrow{ }}$ of the subsequent increments in plasma fibronectin $\vec{\omega}$ concentrations in patients with MCLS, no appropriate explanation can be given at present. It might, for $\stackrel{\circ}{\Omega}$ example, be the result of an overshoot in production 9 in the replenishing process.

We are indebted to Professor Mitsuru Osano, Department of 윽 Pediatrics, Keio University, for his support and encouragement and to Miss Kyoko Shinohara for her technical help.

This work was presented at the 16 th conference of the Japanese dे Society of Pediatric Immunology and Infectious Diseases.

\section{References}

1 Lancer ME, Saba TM. Fibronectin as a co-factor necessary for optimal granulocyte phagocytosis of Staphylococcus aureus. J Reticuloendothel Soc 1981;30:415-24.

2 Rourke FJ, Blumenstock FA, Kaplan JE. Effect of fibronectin fragments on macrophage phagocytosis of gelatinized particles J Immunol 1984;132:1931-6.

${ }^{3}$ Blumenstock FA, Saba TM, Weber P, et al. Biochemical and immunological characterization of human opsonic SB glycoprotein: its identity with cold-insoluble globulin. J Biol Chem 1978;253:4287-91.

${ }^{4}$ Shimizu S, Saito N, Kuratsuji T, et al. Plasma fibronectin concentration in the pediatric population. (in Japanese). Journal of Pediatric Practice (Japan) 1985;48:300-4.

${ }^{5}$ Kawasaki T. Diagnostic guideline of Kawasaki disease (MCLS, muco-cutaneous lymph node syndrome). (in Japanese). Journal of the Japanese Pediatric Society 1984;88:2693-4.

${ }^{6}$ Tanaka N, Sekimoto K, Naoe S. Kawasaki disease: relationship with infantile periarteritis nodosa. Arch Pathol Lab Med 1976;100:81-6.

7 Jaffe EA, Mosher DF. Synthesis of fibronectin by cultured human endothelial cells. $J$ Exp Med 1978;147:1779-91.

Correspondence to Dr Shunichi Shimizu, Keiyu General Hospital, Yokohama 231, Japan.

Received 8 August 1985 